\title{
INTELIGENCIA EMOCIONAL Y PRÁCTICAS RELACIONALES CON LA FAMILIA EN ATENCIÓN TEMPRANA
}

\section{Emotional intelligence and relational practices with family in Early Childhood Intervention}

\author{
María Marco Arenas \\ Universidad de Murcia. Departamento de Métodos de Investigación y Diagnóstico en Educa- \\ ción. Grupo de Investigación en Educación, Diversidad y Calidad \\ maria.marco1@um.es \\ María Cristina SÁNCHEZ LÓPEZ \\ Universidad de Murcia. Departamento de Métodos de Investigación y Diagnóstico en Educa- \\ ción. Grupo de Investigación en Educación, Diversidad y Calidad \\ Francisco Alberto García SÁncheZ \\ Universidad de Murcia. Departamento de Métodos de Investigación y Diagnóstico en Educa- \\ ción. Grupo de Investigación en Educación, Diversidad y Calidad
}

Recepción: 18 de noviembre de 2017

Aceptación definitiva: 26 de enero de 2018

Resumen: La intervención en Atención Temprana está necesariamente dirigida al niño y a su familia. Conseguir mejorar la intervención y colaboración con la familia, a partir de unas adecuadas prácticas relacionales, es una preocupación actual en la disciplina. Nuestro objetivo fue comprobar si determinadas habilidades de inteligencia emocional, en los profesionales, pueden estar vinculadas con un mayor o menor ejercicio de prácticas relacionales con la familia. Participaron 420 profesionales, 25 hombres y 387 mujeres, de 13 comunidades autónomas de España. Cumplimentaron dos instrumentos: Trait Meta-MoodScale (TMMS-24), del grupo de investigación de Salovey y Mayer, traducida por Fernández-Berrocal y Extremera (2006); Inventario sobre Práctica Profesional en Atención Temprana (IPPAT), creado al efecto. Los resultados demuestran que los profesionales de Atención Temprana realizan prácticas relacionales en sus intervenciones y poseen altos niveles de habilidades emocionales. Con todo, 
encontramos diferencias significativas en estas prácticas relacionales en función de sus puntuaciones en las habilidades de inteligencia emocional. Los resultados se discuten también en términos de sus implicaciones de cara a la formación de futuros profesionales en Atención Temprana.

Palabras clave: Atención Temprana; inteligencia emocional; prácticas relacionales; desarrollo profesional.

AвSTRAct: The work in Early Intervention is necessarily directed to the child and his family. To improve the intervention and collaboration with the family, from appropriate relational practices, is a current concern in the discipline. The purpose of the study was to verify whether certain emotional intelligence skills, in professionals, may be linked to relational practices within families. A group of 420 professionals (composed of 25 men and 387 women) from 13 autonomous Spanish communities participated in the study. They completed two instruments: Trait Meta-MoodScale (TMMS-24) of the Salovey and Mayer research group, translated by Fernández-Berrocal and Extremera (2006); Inventory on Professional Practice in Early Intervention (IPPAT), created for this purpose. The results show that early intervention professionals perform relational practices in their interventions and possess high levels of emotional skills. However, we found significant differences in these relational practices based on their scores on the skills of emotional intelligence. The results are also discussed in terms of their implications for the training of future professionals in Early Childhood Intervention.

KEY WORDS: Early Intervention; emotional intelligence; relational practices; professional development.

\section{Introducción}

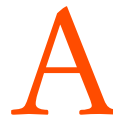

L Desarrollar la intervención en Atención Temprana, el profesional debe saber interaccionar con el niño de 0 a 6 años, con alteraciones en su desarrollo o riesgo de padecerlas. Pero debe, además, saber interactuar también con unos padres inicialmente afectados y desorientados por la situación que vive su niño (GAT, 2000, 2011). Sus objetivos serán el desarrollo del niño y la mejora de la calidad de vida de la familia en su conjunto. Para conseguirlos, este profesional tendrá que llevar a cabo, con los padres, tanto unas prácticas participativas como relacionales (Dunst, Boyd, Trivette y Hamby, 2002; Espe-Sherwindt, 2008; García-Sánchez, Escorcia, Sánchez-López, Orcajada y Hernández, 2014). Las prácticas que denominamos participativas son aquellas que llevan a fomentar una mayor participación e implicación directa de la familia en la intervención y en la toma de decisiones en torno a sus objetivos. Estas prácticas participativas serán más o menos intensas, según el profesional esté trabajando desde un paradigma de intervención ambulatoria o centrado en la familia. Desde una intervención ambulatoria, el profesional suele posicionarse como experto, tomando decisiones y realizando directamente la intervención. Desde un paradigma de intervención centrada en la familia, el profesional debe centrarse en desarrollar una 
interacción colaborativa con la familia y potenciar su competencia para favorecer el desarrollo del niño, aprovechando oportunidades de aprendizaje contextualizadas. Por su parte, las prácticas que denominamos relacionales son más independientes de cuál sea el posicionamiento teórico-práctico del profesional (Espe-Sherwindt, 2008). Todos los profesionales de Atención Temprana tendrán que desarrollar, siempre, unas prácticas relacionales, que le permitan establecer, con las familias, las imprescindibles alianzas mínimas terapéuticas o de intervención y colaboración.

Dentro de lo que podemos incluir en esas prácticas relacionales, distintos trabajos han resaltado la necesidad de acompañar a la familia en el duelo que inicialmente suelen experimentar por el nacimiento de su niño con discapacidad, contribuyendo y ayudándoles a superarlo (DeMarle y le Roux, 2001; Mendieta, 2005). Igualmente, se ha descrito la necesidad de ayudarles a manejar el estrés que puede acompañar a los padres en la crianza de un niño con discapacidad (Azad, Blacher y Marcoulides, 2013; Barnett, Clements, Kaplan-Estrin y Fialka, 2003; Peer y Hillman, 2014). Otros trabajos han resaltado la necesidad de establecer unos lazos de confianza, corresponsabilidad e, incluso, una relación emocional controlada, que favorezca alianzas de trabajo productivas entre profesional-familia-niño (Castellanos, García-Sánchez, Mendieta, Gómez y Rico, 2003; Dalmau-Montala et al., 2017). También cuando el profesional actúa como un mero terapeuta rehabilitador del paciente, su efectividad se ha asociado a las relaciones terapeuta-paciente establecidas. Se entienden necesarias unas relaciones que permitan entender la experiencia subjetiva del paciente y, a la vez, transmitirle sensaciones de aceptación y respeto (Fernández-Zúñiga y Marcos de León, 2008).

Además, no debemos olvidar que el profesional de Atención Temprana debe trabajar en equipo, colaborando estrechamente con otros profesionales de su propio CDIAT. Incluso con profesionales ajenos a él, pero que también atienden al niño y su familia desde el ámbito educativo o sanitario (Arnaldos et al., 2000; GAT, 2000; King et al., 2009). También, en estas interacciones, habrán de ponerse en marcha prácticas relacionales que contribuyan a lograr las metas buscadas.

Cuando hablamos de que el profesional ponga en marcha prácticas relacionales, le estamos pidiendo que utilice un conjunto de competencias, innatas o adquiridas, relacionadas estrechamente con la inteligencia emocional. Estas competencias incluyen elementos de buena capacidad de comunicación, como escucha activa, respeto y empatía, junto con adecuada competencia profesional, que transmita sensación de confianza y compromiso (Blue-Banning, Summers, Frankland, Nelson y Beegle, 2004; DalmauMontala et al., 2017; Dempsey y Dunst, 2004; Dunst et al., 2002; Espe-Sherwindt, 2008; Ginè, Gràcia, Vilaseca y Balcells, 2008; Turnbull, Turnbull, Erwin y Soodak, 2006; Wilson y Dunst, 2005). Los profesionales en Atención Temprana deben desarrollar competencias asociadas o dirigidas a la interacción positiva y colaboración con la familia y con otros profesionales. Especialmente con la familia, deben desarrollar un esmerado trato adecuado, manteniendo comportamientos interpersonales apropiados (Perpiñan, 2009; Stoner, Meadan y Angell, 2013). La comunicación debe ser fluida y efectiva, cuando las familias expresan sus debilidades y fortalezas (Banerjee y Luckner, 2014; Turnbull et al., 2006). En torno a la diversidad cultural, a las opiniones y los 
deseos de cada familia, el profesional debe respetar todas las situaciones o creencias, mostrando capacidad de adaptarse a la situación sociofamiliar del niño antes y durante la intervención (Espe-Sherwindt, 2008; Sawyer y Campell, 2012).

En Atención Temprana, al igual que ocurre en otros ámbitos de estudio o de trabajo (Cantero, 2012), se están demandando trabajadores competentes, que destaquen tanto en habilidades cognitivas y profesionales, como en competencias intrapersonales y sociales. Para ello, va a ser esencial que el profesional cuente con una adecuada inteligencia emocional, entendida como aquella inteligencia que va más allá de las competencias meramente cognitivas, al tener en cuenta, también, la capacidad personal de entender, utilizar y regular las emociones que aparecen en toda interacción humana. Capacidades que son esenciales para la autogestión personal y del trabajo con otros y para el desarrollo de una adecuada competencia social (Côté, 2014; Côté y Hideg, 2011; Goleman 1996; Lopes, 2016). Los profesionales deben poseer distintas competencias socioemocionales como la autoconfianza, el autocontrol, la paciencia, la motivación, la empatía... para establecer buenas relaciones tanto en su vida personal como laboral (Bisquerra y Pérez, 2007). En este sentido, ha llegado a argüirse que las personas emocionalmente inteligentes son más felices, se sienten más satisfechas y con mayores índices de bienestar. Por tanto, poseen mejores relaciones interpersonales y sociales, tanto en el entorno personal como profesional (Fernández-Berrocal, 2010; Fernández-Berrocal y Extremera, 2009). También el adecuado trabajo en equipo está marcado por las capacidades de inteligencia emocional de los profesionales que los integran (Kaplan y Welter, 2001; Jordan y Ashkanasy, 2006).

El presente estudio plantea el objetivo general de comprobar si determinadas habilidades de inteligencia emocional de los profesionales de Atención Temprana pueden estar vinculadas con un mayor o menor ejercicio de prácticas relacionales en las interacciones con la familia. Para ello, primero analizaremos el volumen de prácticas relacionales que desarrollan los profesionales, a partir de su autovaloración ante los ítems presentados. En segundo lugar, analizaremos si la mayor o menor presencia, en los profesionales, de las tres habilidades emocionales medidas (atención, claridad y reparación emocional) influye en la identificación de un mayor o menor volumen de prácticas relacionales. En tercer lugar, analizaremos si esa influencia se da en mayor o en menor medida en alguna de las dimensiones planteadas en torno a las prácticas relacionales identificadas. Por último, analizaremos si otras variables, como la edad del profesional, su formación y su experiencia o veteranía en Atención Temprana, influyen también en la valoración que los profesionales hacen de las prácticas relacionales desarrolladas con las familias con las que intervienen en Atención Temprana.

\section{Método}

\subsection{Participantes}

La población objeto de la investigación fue seleccionada a través de un muestreo no probabilístico intencional. Se contactó telefónicamente con 250 CDIAT de toda 
España, a todos los cuales se enviaron, por correo electrónico, los dos instrumentos a cumplimentar. Devolvieron los instrumentos cumplimentados un total de 91 CDIAT (un 36,4\% de los contactados), distribuidos en 13 comunidades autónomas.

Los CDIAT que participaron enviaron finalmente un total de 420 cuestionarios cumplimentados. Entre los perfiles profesionales de los profesionales que los cumplimentaron encontramos Fisioterapia (78), Logopedia (97), Trabajo Social (36), Psicología (104), Pedagogía (55), Maestro de Educación Especial (19), Maestro de Educación Infantil (9) y Neuropediatría (2). En los profesionales participantes se registró un amplio rango de edades (21 a 62 años), si bien el 77.7\% presentó edades que oscilaban entre 21 y 41 años, siendo la media de la edad de 36 años. Se observa un predominio del género femenino (387 mujeres frente a 25 hombres, 8 profesionales no indicaron género). De los profesionales participantes, el 26.2\% tiene máster en Atención Temprana. En cuanto a los años trabajados en Atención Temprana, el 65\% lleva entre cinco y diez años trabajando en este campo.

\subsection{Procedimiento}

Durante el curso 2016-2017, tras llamada telefónica a la dirección de cada CDIAT, se enviaron los dos instrumentos a cumplimentar. La dirección del centro o la figura responsable nombrada por el CDIAT se encargaba de recopilar los cuestionarios cumplimentados y devolverlos al correo electrónico de origen. Algunos centros prefirieron imprimir los inventarios y remitirlos posteriormente por correo postal.

\subsection{Instrumentos}

Para conocer el desarrollo de prácticas relacionales y participativas por parte del profesional, se utilizó el Inventario sobre Práctica Profesional en Atención Temprana (IPPAT). Esta herramienta fue creada para la investigación, a través de un proceso sistemático de identificación de ítems y dimensiones de interés para conocer las prácticas realizadas con la familia. Se consultó para ello diferente bibliografía. Especialmente trabajos clásicos, definitorios de los componentes relacionales y participativos en las prácticas de Atención Temprana (Dunst, 2002; Dunst y Trivette, 1996); junto con trabajos que delimitaban ítems para identificar algunas de estas prácticas (Escorcia, García-Sánchez, Sánchez-López y Hernández-Pérez, 2016). La primera versión del instrumento fue sometida a un procedimentado juicio de experto. En él participaron 8 profesionales: 3 profesores de universidad del área de Métodos de Investigación y Diagnóstico en Educación y 5 profesionales de Atención Temprana, con una media de más de 20 años de experiencia laboral. En dicho juicio de expertos se valoró, por separado y en una escala de 4 puntos, la idoneidad del contenido de los ítems, su grado de claridad y representatividad, atendiendo a los constructos sobre los que se quería indagar. También se valoraron aspectos generales del instrumento, como su presentación y delimitación de características 
del encuestado. El instrumento final quedó constituido por 45 ítems, aleatoriamente distribuidos. De ellos, 15 ítems estaban referidos a prácticas relacionales, 26 a prácticas participativas y 4 al trabajo ambulatorio. En los ítems dedicados a las prácticas relaciones del profesional, cuyos resultados son los que se presentan en este estudio, se repartieron en 5 dimensiones teóricas, que pueden inferirse de la literatura consultada (Dunst, 2002; Dunst y Trivette, 1996; Escorcia et al., 2016): Interacción profesional y familia (5 ítems), Trato con la familia (5 ítems), Comunicación (3 ítems), Respeto de creencias y valores (1 ítem) y Reconocimiento de habilidades y fortalezas (1 ítem). La redacción específica de los ítems queda recogida en las tablas presentadas en el apartado de resultados de este trabajo. La escala de valoración de cada ítem, en referencia a la práctica habitual del profesional, es de 5 puntos, que corresponden a las etiquetas verbales de Nunca, Casi nunca, Algunas veces, Casi siempre y Siempre. La fiabilidad alcanzada en estos ítems sobre prácticas relacionales fue de un alfa de Cronbach de .721, siendo .911 el valor alcanzado para el instrumento en su conjunto. No se ha llevado a cabo un estudio psicométrico de la adecuación de la distribución de los ítems en las dimensiones planteadas. Por ello, se analizarán los resultados para cada ítem por separado, dado que el juicio de expertos avala su utilidad para el propósito del estudio.

Para la evaluación de la Inteligencia Emocional se utilizó la escala Trait MetaMood Scale (TMMS-24), adaptada por Fernández-Berrocal, Extremera y Ramos (2004). Este instrumento es utilizado frecuentemente en diferentes investigaciones de ámbito educativo y sanitario (Espinoza, Sanhueza, Ramírez y Sáez, 2015; Extremera, Fernández-Berrocal y Durán, 2003). Evalúa el metaconocimiento de los estados emocionales a través de 24 ítems distribuidos en tres habilidades emocionales con 8 ítems cada una: Atención emocional (capacidad de sentir y expresar sentimientos), Claridad emocional (capacidad para reconocer nuestros estados emocionales) y Reparación emocional (capacidad de afrontar y canalizar nuestros estados emocionales). El instrumento se cumplimenta en función del grado de acuerdo para cada ítem, a modo de escala Likert con puntuaciones desde $1=$ Nada de acuerdo, $2=$ Algo de acuerdo, 3 = Bastante de acuerdo, $4=$ Muy de acuerdo y $5=$ Totalmente de acuerdo. La escala ofrece los siguientes valores para su corrección e interpretación: Poca Atención ( $<24$ en mujeres; < 21 en hombres), adecuada atención (25-35 en mujeres; 22-32 en hombres) y demasiada atención ( $>36$ en mujeres; $>33$ en hombres). Para las habilidades emocionales Claridad y Reparación hay menos diferencias entre géneros y se pueden considerar los siguientes valores: debe mejorar $(<23)$, adecuada (24-35) y excelente ( $>36)$.

Esta escala tiene una rápida y fácil administración. Además, cuenta con una excelente fiabilidad en estudios previos (Espinoza et al., 2015; Extremera y FernándezBerrocal, 2004). Con la muestra de este estudio, la fiabilidad alcanzada por el instrumento en su conjunto fue de un alfa de Cronbach de .89. La fiabilidad alcanzada por las tres subescalas fue de .86 para Atención Emocional, .89 para Claridad Emocional y .87 para Reparación Emocional. 


\subsection{Análisis de los datos}

Los datos se analizaron tanto de forma descriptiva, a través del análisis de medias y desviaciones típicas, como inferencial. Para el análisis de diferencias entre medias se utilizó la prueba t-Student para muestras independientes, calculándose el estadístico d de Cohen para determinar el tamaño del efecto. El programa SPSS, en su versión 19.0, fue utilizado para el análisis estadístico de los datos.

\section{Resultados}

En respuesta al primer objetivo del estudio, en conjunto, los ítems planteados sobre prácticas relacionales alcanzan una media de 4.13 puntos $(\mathrm{dt}=.32)$, lo que equivale a una etiqueta verbal de Casi Siempre en las respuestas de los profesionales.

La respuesta al segundo objetivo puede verse en la Tabla 1. Se encontraron diferencias estadísticamente significativas al comparar las puntuaciones directas de los profesionales, en el conjunto de ítems utilizados sobre prácticas relacionales, una vez que fueron divididos en dos grupos en función de que presenten las puntuaciones medias más altas (tercer cuartil) y más bajas (primer cuartil) en las tres habilidades de inteligencia emocional del TMMS-24: Atención Emocional, Claridad Emocional y Reparación Emocional. Los resultados alcanzados por la prueba de estimación del tamaño del efecto nos indican que estos tamaños tienen una magnitud mediana.

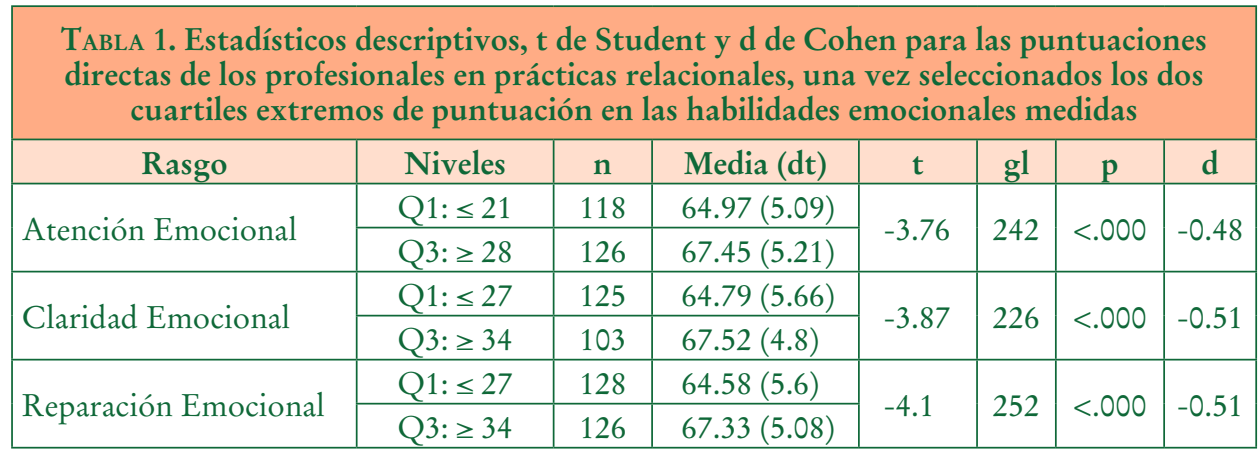

Las características de los profesionales de los dos grupos de puntuaciones más altas y bajas en las habilidades de inteligencia emocional se presentan en la Tabla 2. Se observa que presentan valores equiparables en edad media, distribución por sexo, veteranía en Atención Temprana y distribución por titulación.

Para responder al tercer objetivo del estudio y profundizar en la comprensión de la posible influencia de las habilidades de inteligencia emocional en las dimensiones de prácticas relacionales planteadas, presentamos a continuación los resultados obtenidos en los ítems en cada una de esas dimensiones. 
INTELIGENCIA EMOCIONAL Y PRÁCTICAS RELACIONALES CON LA FAMILIA EN ATENCIÓN TEMPRANA MARÍA MARCO ARENAS, MARÍA CRISTINA SÁNCHEZ LÓPEZ Y FRANCISCO ALBERTO GARCÍA SÁNCHEZ

TABLA 2. Estadísticos descriptivos de las características de los profesionales integrados
en los cuartiles extremos de puntuación en las tres habilidades emocionales medidas

\begin{tabular}{|l|c|c|c|c|c|c|}
\hline & \multicolumn{2}{|c|}{$\begin{array}{c}\text { Atención } \\
\text { Emocional }\end{array}$} & \multicolumn{2}{c|}{$\begin{array}{c}\text { Claridad } \\
\text { Emocional }\end{array}$} & \multicolumn{2}{c|}{$\begin{array}{c}\text { Reparación } \\
\text { Emocional }\end{array}$} \\
\cline { 2 - 7 } & Q1 & Q3 & Q1 & Q3 & Q1 & Q3 \\
\hline Edad media (dt) & $37.0(8.86)$ & $35.83(8.25)$ & $36.92(8.74)$ & $35.65(8.66)$ & $37.07(8.86)$ & $34.96(8.23)$ \\
\hline Años experiencia (dt) & $9.02(7.38)$ & $8.5(6.43)$ & $9.18(7.17)$ & $8.7(7.04)$ & $9.63(7.72)$ & $7.78(6.42)$ \\
\hline Varones (\%) & 6.0 & 4.9 & 4.9 & 6.0 & 7.1 & 4.1 \\
\hline Mujeres (\%) & 94.0 & 95,1 & 95.1 & 94.0 & 92.9 & 95.9 \\
\hline Fisioterapia (\%) & 23.6 & 17.5 & 23.9 & 15.2 & 19.2 & 23.5 \\
\hline Logopedia (\%) & 23.6 & 22.5 & 26.5 & 23.2 & 25.8 & 28.6 \\
\hline Trabajo Social (\%) & 7.3 & 10.8 & 12.0 & 10.1 & 10.8 & 5.9 \\
\hline Psicología (\%) & 25.5 & 27.5 & 21.4 & 26.3 & 26.7 & 16.8 \\
\hline Pedagogía (\%) & 12.7 & 15.0 & 11.1 & 18.2 & 12.5 & 18.5 \\
\hline Maestro Ed. Especial (\%) & 3.6 & 4.2 & 3.4 & 4.0 & 3.3 & 3.4 \\
\hline Maestro Ed. Infantil (\%) & 2.7 & 1.7 & 0.9 & 3.0 & 0.8 & 2.5 \\
\hline Neuropediatría(\%) & 0.9 & 0.8 & 0.9 & - & 0.8 & 0.8 \\
\hline
\end{tabular}

En la Tabla 3 se muestran, atendiendo a los resultados en la habilidad de Atención Emocional del TMMS-24, las medias y desviaciones típicas de las respuestas de los profesionales en los ítems sobre prácticas relacionales del IPPAT. Se consideraron las respuestas de los 118 profesionales con puntuaciones $\leq 21$ en la habilidad de Atención Emocional (Q1) y los 126 profesionales con puntuaciones $\geq 28$ en dicha habilidad (Q3). Además, se presentan los resultados de la prueba t de Student para el contraste de las medias y el estadístico d de Cohen para valorar el tamaño del efecto.

\begin{tabular}{|c|c|c|c|c|c|c|}
\hline & $\mathrm{Q}$ & Media (dt) & $\mathrm{t}$ & $\mathrm{gl}$ & $\mathrm{p}$ & $\mathrm{d}$ \\
\hline \multicolumn{7}{|l|}{ Interacción profesional-familia } \\
\hline \multirow{2}{*}{ Me presento a la familia como un apoyo } & Q1 & $4.33(.84)$ & \multirow{2}{*}{-2.09} & \multirow{2}{*}{242} & \multirow{2}{*}{.038} & \multirow{2}{*}{-0.27} \\
\hline & Q3 & $4.55(.79)$ & & & & \\
\hline \multirow{2}{*}{$\begin{array}{l}\text { Promuevo un clima de confianza en las interacciones con el } \\
\text { cuidador principal }\end{array}$} & Q1 & $4.74(.44)$ & \multirow{2}{*}{.74} & \multirow{2}{*}{242} & \multirow{2}{*}{.463} & \multirow{2}{*}{0.1} \\
\hline & Q3 & $4.69(.54)$ & & & & \\
\hline \multirow{2}{*}{ Pregunto por el estado del niño al comenzar la sesión } & Q1 & $4.32(.83)$ & \multirow{2}{*}{-1.11} & \multirow{2}{*}{242} & \multirow{2}{*}{.268} & \multirow{2}{*}{-0.15} \\
\hline & Q3 & $4.44(.79)$ & & & & \\
\hline \multirow{2}{*}{ Respeto las creencias de la familia durante la intervención } & Q1 & $4.43(.78)$ & \multirow{2}{*}{-1.66} & \multirow{2}{*}{242} & \multirow{2}{*}{.098} & \multirow{2}{*}{-0.22} \\
\hline & Q3 & $4.58(.60)$ & & & & \\
\hline \multirow{2}{*}{$\begin{array}{l}\text { Me adapto a las condiciones del entorno del niño sin modi- } \\
\text { ficarlo a mi estilo }\end{array}$} & Q1 & $3.68(.97)$ & \multirow{2}{*}{-2.35} & \multirow{2}{*}{241} & \multirow{2}{*}{.019} & \multirow{2}{*}{-0.3} \\
\hline & Q3 & $3.96(.92)$ & & & & \\
\hline
\end{tabular}


INTELIGENCIA EMOCIONAL Y PRÁCTICAS RELACIONALES CON LA FAMILIA EN ATENCIÓN TEMPRANA MARÍA MARCO ARENAS, MARÍA CRISTINA SÁNCHEZ LÓPEZ Y FRANCISCO ALBERTO GARCÍA SÁNCHEZ

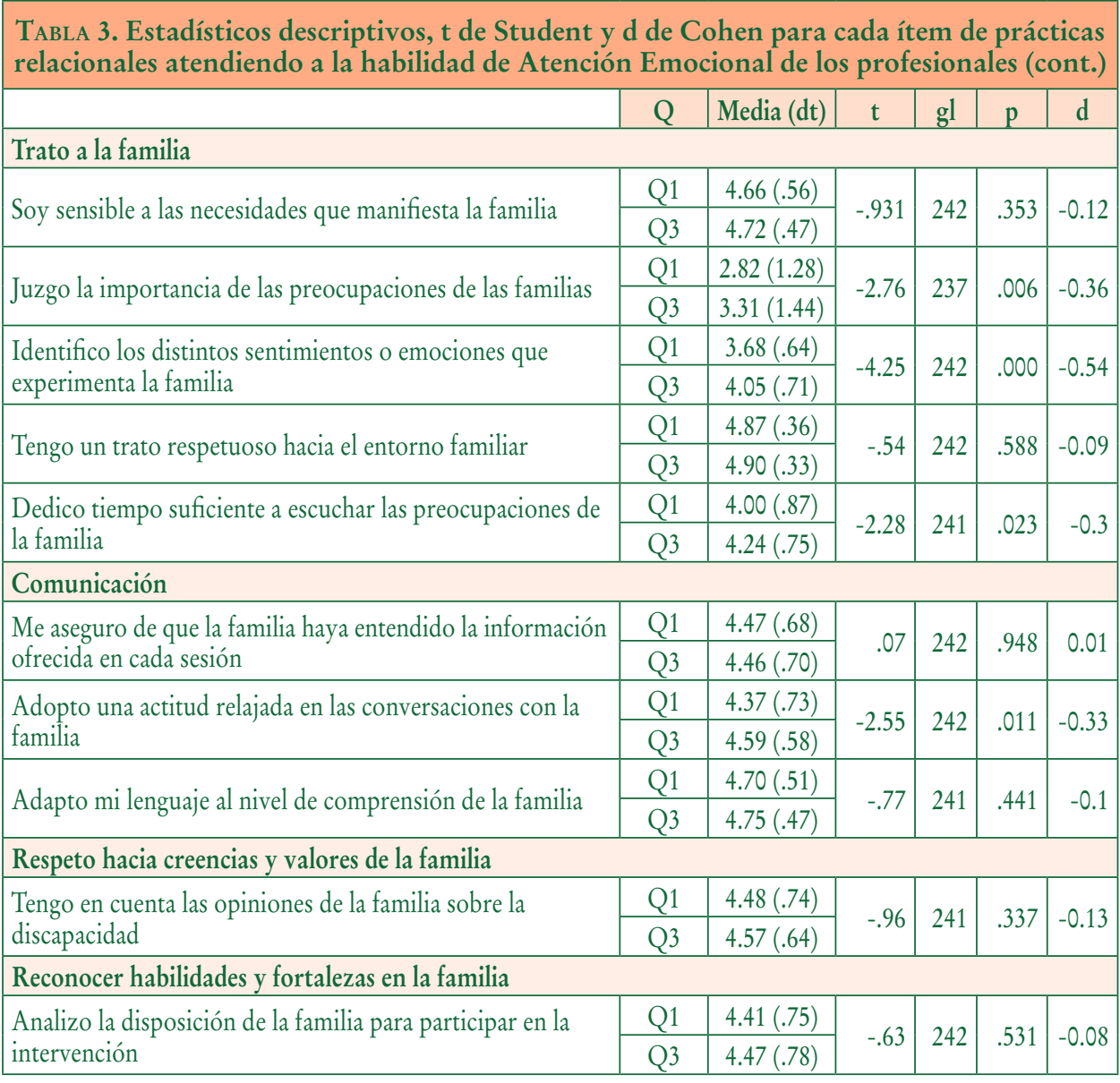

En la valoración que los profesionales hacen de las distintas propuestas de prácticas relacionales se encontraron seis ítems en los que aquellos con una habilidad de Atención Emocional más baja (Q1) presentan unas puntuaciones medias significativamente más bajas que sus compañeros con puntuaciones más altas en esa habilidad de inteligencia emocional (Q3). Esto supone que la habilidad de Atención Emocional está afectando al $40 \%$ de los ítems planteados de prácticas relacionales.

En concreto, los profesionales situados en el tercer cuartil en la habilidad de Atención Emocional puntúan significativamente más alto que sus compañeros del cuartil uno en dos ítems de la dimensión de prácticas relacionales que hemos denominado interacción entre profesional y familia ("me presento a la familia como un apoyo" y "me adapto a las condiciones del entorno del niño sin modificarlas a mi estilo"); en tres ítems de la dimensión de trato a la familia ("juzgo la importancia de las preocupaciones de la familia”, “identifico los distintos sentimientos o emociones que 
experimenta la familia” y "dedico tiempo suficiente a escuchar las preocupaciones de la familia”); y en un ítem de la dimensión de comunicación (“adopto una actitud relajada en las conversaciones con la familia”). La habilidad de Atención Emocional no ocasiona diferencias significativas en las dimensiones de respeto bacia las creencias $y$ valores de la familia y reconocimiento de habilidades y fortalezas en la familia. Los resultados de la prueba d de Cohen indican que el tamaño del efecto es, en todos los casos, pequeño (entre -0.27 y -0.36), alcanzando un tamaño moderado en el caso del ítem en el que planteamos que el profesional "identifica los distintos sentimientos o emociones que experimenta la familia” (-0.54).

En la Tabla 4 se muestran, atendiendo a los resultados en la habilidad de Claridad Emocional del TMMS-24, las medias y las desviaciones típicas de las respuestas de los profesionales en los ítems sobre prácticas relacionales del IPPAT. Se consideraron las respuestas de los 125 profesionales con puntuaciones $\leq 27$ en la habilidad Claridad Emocional (Q1) y los 103 profesionales con puntuaciones $\geq 34$ en dicha habilidad (Q3). Además, se presentan los resultados de la prueba t de Student para el contraste de las medias y el estadístico d de Cohen para valorar el tamaño del efecto.

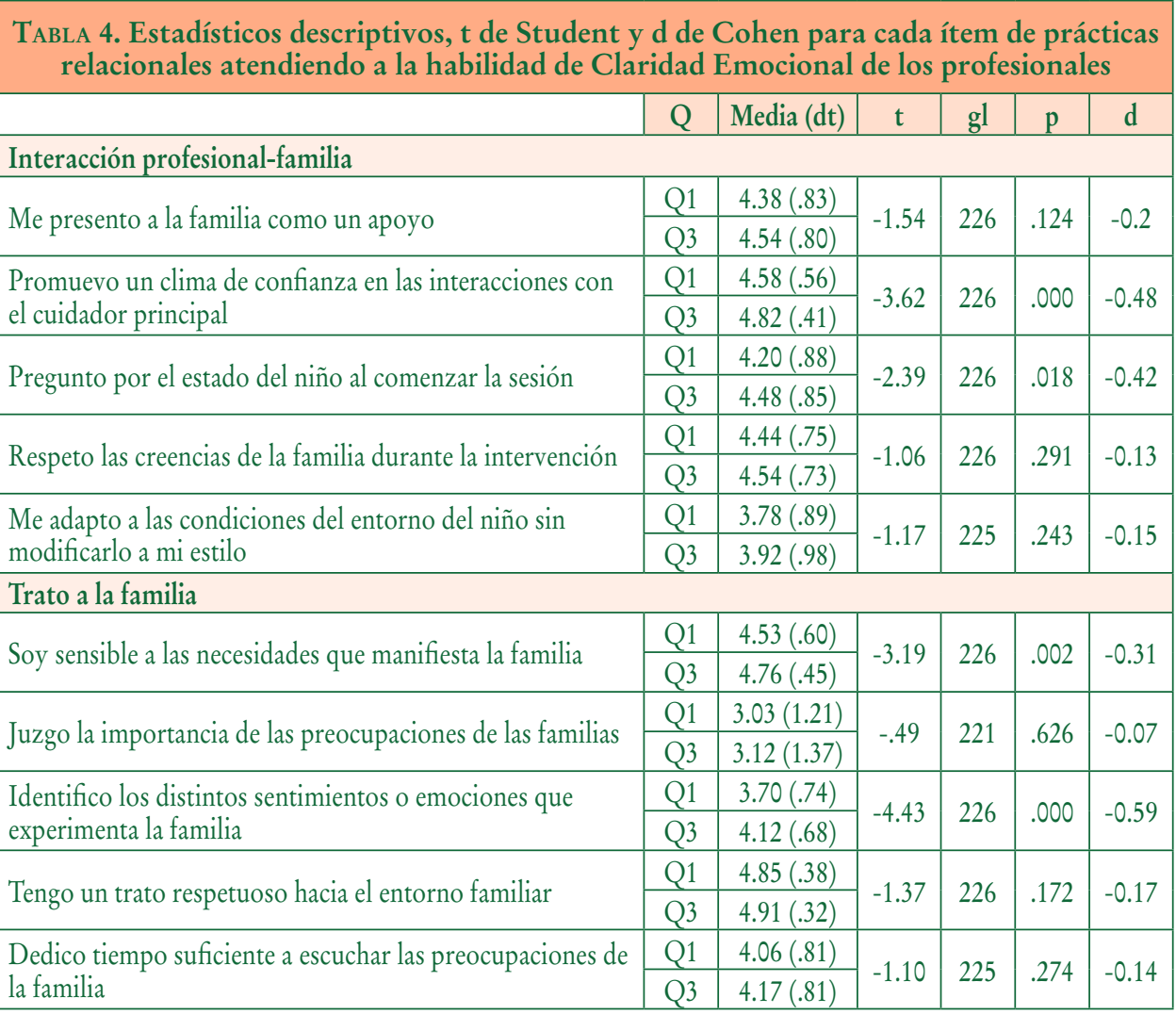


INTELIGENCIA EMOCIONAL Y PRÁCTICAS RELACIONALES CON LA FAMILIA EN ATENCIÓN TEMPRANA MARÍA MARCO ARENAS, MARÍA CRISTINA SÁNCHEZ LÓPEZ Y FRANCISCO ALBERTO GARCÍA SÁNCHEZ

\begin{tabular}{|c|c|c|c|c|c|c|}
\hline & $\mathrm{Q}$ & Media (dt) & $\mathrm{t}$ & $\mathrm{gl}$ & $\mathrm{p}$ & $\mathrm{d}$ \\
\hline \multicolumn{7}{|l|}{ Comunicación } \\
\hline \multirow{2}{*}{$\begin{array}{l}\text { Me aseguro de que la familia haya entendido la } \\
\text { información ofrecida en cada sesión }\end{array}$} & Q1 & $4.35(.75)$ & \multirow{2}{*}{-2.55} & \multirow{2}{*}{226} & \multirow{2}{*}{.011} & \multirow{2}{*}{-0.34} \\
\hline & Q3 & $4.59(.65)$ & & & & \\
\hline \multirow{2}{*}{$\begin{array}{l}\text { Adopto una actitud relajada en las conversaciones con la } \\
\text { familia }\end{array}$} & Q1 & $4.38(.74)$ & \multirow{2}{*}{-3.41} & \multirow{2}{*}{226} & \multirow{2}{*}{.001} & \multirow{2}{*}{-0.46} \\
\hline & Q3 & $4.68(.53)$ & & & & \\
\hline \multirow{2}{*}{ Adapto mi lenguaje al nivel de comprensión de la familia } & Q1 & $4.69(.50)$ & \multirow{2}{*}{-2.21} & \multirow{2}{*}{225} & \multirow{2}{*}{.028} & \multirow{2}{*}{-0.28} \\
\hline & Q3 & $4.82(.41)$ & & & & \\
\hline \multicolumn{7}{|l|}{ Respeto hacia creencias y valores de la familia } \\
\hline \multirow{2}{*}{$\begin{array}{l}\text { Tengo en cuenta las opiniones de la familia sobre la } \\
\text { discapacidad }\end{array}$} & Q1 & $4.39(.74)$ & \multirow{2}{*}{-2.88} & \multirow{2}{*}{225} & \multirow{2}{*}{.004} & \multirow{2}{*}{-0.39} \\
\hline & Q3 & $4.65(.56)$ & & & & \\
\hline \multicolumn{7}{|l|}{ Reconocer habilidades y fortalezas en la familia } \\
\hline \multirow{2}{*}{$\begin{array}{l}\text { Analizo la disposición de la familia para participar en la } \\
\text { intervención }\end{array}$} & Q1 & $4.33(.80)$ & \multirow{2}{*}{-1.11} & \multirow{2}{*}{226} & \multirow{2}{*}{.267} & \multirow{2}{*}{-0.15} \\
\hline & Q3 & $4.45(.80)$ & & & & \\
\hline
\end{tabular}

En la valoración que los profesionales hacen de las distintas propuestas de prácticas relacionales planteadas se encontraron ocho ítems en los que los profesionales con una habilidad de Claridad Emocional más baja (Q1) presentan unas puntuaciones medias significativamente más bajas que sus compañeros con puntuaciones más altas en esa habilidad de inteligencia emocional (Q3). Esto supone que la habilidad de Claridad Emocional está afectando al $53.33 \%$ de los ítems de prácticas relacionales planteados.

En concreto, los profesionales situados en el tercer cuartil en la habilidad de Claridad Emocional puntúan significativamente más alto que sus compañeros del cuartil uno en dos ítems de la dimensión de prácticas relacionales denominada interacción entre profesional y familia ("promuevo un clima de confianza en las interacciones con el cuidador principal” y "pregunto por el estado del niño al comenzar la sesión”); en otros dos ítems de la dimensión de trato a la familia ("soy sensible a las necesidades que manifiesta la familia" e "identifico los distintos sentimientos o emociones que experimenta la familia”); en tres ítems de la dimensión de comunicación ("me aseguro de que la familia haya entendido la información ofrecida en cada sesión”, "adopto una actitud relajada en las conversaciones con la familia” y "adapto mi lenguaje al nivel de comprensión de la familia”); y en el ítem de la dimensión de respeto hacia las creencias y valores de la familia ("tengo en cuenta las opiniones de la familia sobre la discapacidad”). La habilidad de Claridad Emocional no ocasiona diferencias significativas en la dimensión de reconocimiento de babilidades y fortalezas en la familia. Los resultados de la prueba d de Cohen indican que el tamaño del efecto es, en todos los casos, entre pequeño y moderado. Obtiene el tamaño más bajo el ítem que plantea que el profesional adapta su lenguaje al nivel de comprensión de la familia (-0.28). Por su parte, el efecto alcanza un tamaño moderado en tres ítems: "promuevo un clima 
de confianza en las interacciones con el cuidador principal" (-0.48), "identifico los distintos sentimientos o emociones que experimenta la familia" (-0.59) y "adopto una actitud relajada en las conversaciones con la familia" (-0.46).

En la Tabla 5 se muestran, atendiendo a los resultados en la habilidad de Reparación Emocional del TMMS-24, las medias y las desviaciones típicas de las respuestas de los profesionales en los ítems sobre prácticas relacionales del IPPAT. Se consideraron las respuestas de los 128 profesionales con puntuaciones $\leq 27$ en la habilidad Reparación Emocional (Q1) y los 126 profesionales con puntuaciones $\geq 34$ en dicha habilidad (Q3). Además, se presentan los resultados de la prueba t de Student para el contraste de las medias y el estadístico d de Cohen para valorar el tamaño del efecto.

\begin{tabular}{|c|c|c|c|c|c|c|}
\hline & $\mathrm{Q}$ & Media (dt) & $\mathrm{t}$ & $\mathrm{gl}$ & $\mathrm{p}$ & $\mathrm{d}$ \\
\hline \multicolumn{7}{|l|}{ Interacción profesional-familia } \\
\hline \multirow{2}{*}{ Me presento a la familia como un apoyo } & Q1 & $4.30(.88)$ & \multirow{2}{*}{-3.13} & \multirow{2}{*}{252} & \multirow{2}{*}{.002} & \multirow{2}{*}{-0.38} \\
\hline & Q3 & $4.60(.66)$ & & & & \\
\hline \multirow{2}{*}{$\begin{array}{l}\text { Promuevo un clima de confianza en las interacciones con el } \\
\text { cuidador principal }\end{array}$} & Q1 & $4.56(.59)$ & \multirow{2}{*}{-4.62} & \multirow{2}{*}{252} & \multirow{2}{*}{.000} & \multirow{2}{*}{-0.58} \\
\hline & Q3 & $4.85(.38)$ & & & & \\
\hline \multirow{2}{*}{ Pregunto por el estado del niño al comenzar la sesión } & Q1 & $4.12(.88)$ & \multirow{2}{*}{-3.51} & \multirow{2}{*}{252} & \multirow{2}{*}{.001} & \multirow{2}{*}{-0.43} \\
\hline & Q3 & $4.49(.82)$ & & & & \\
\hline \multirow{2}{*}{ Respeto las creencias de la familia durante la intervención } & Q1 & $4.45(.76)$ & \multirow{2}{*}{-1.28} & \multirow{2}{*}{252} & \multirow{2}{*}{.202} & \multirow{2}{*}{-0.16} \\
\hline & Q3 & $4.57(.71)$ & & & & \\
\hline \multirow{2}{*}{$\begin{array}{l}\text { Me adapto a las condiciones del entorno del niño sin } \\
\text { modificarlo a mi estilo }\end{array}$} & Q1 & $3.77(.95)$ & \multirow{2}{*}{-1.05} & \multirow{2}{*}{250} & \multirow{2}{*}{.296} & \multirow{2}{*}{-0.13} \\
\hline & Q3 & $3.90(1.01)$ & & & & \\
\hline \multicolumn{7}{|l|}{ Trato a la familia } \\
\hline \multirow{2}{*}{ Soy sensible a las necesidades que manifiesta la familia } & Q1 & $4.53(.57)$ & \multirow{2}{*}{-3.04} & \multirow{2}{*}{252} & \multirow{2}{*}{.003} & \multirow{2}{*}{-0.38} \\
\hline & Q3 & $4.73(.46)$ & & & & \\
\hline Juzon la imnortoncin de las nrencumaciones de las familins & Q1 & $2.83(1.22)$ & 190 & 248 & 059 & 023 \\
\hline Juzgo la importancia de las preocupaciones de las tamilias & Q3 & $3.15(1.50)$ & -1.90 & 248 & .059 & -0.23 \\
\hline Identifico los distintos sentimientos o emociones que & Q1 & $3.77(.77)$ & 283 & 252 & 005 & 035 \\
\hline experimenta la familia & Q3 & $4.02(.64)$ & $\mid-2.83$ & 232 & .005 & $\mid-0.35$ \\
\hline Tenoo un trato resnetuoso hucin el entorno familiar & Q1 & $4.81(.47)$ & - 200 & 252 & 045 & -025 \\
\hline etuoso hacia el entorno familiar & Q3 & $4.91(.31)$ & -2.02 & 252 & .045 & $\mid-0.25$ \\
\hline Dedico tiempo suficiente a escuchar las preocupaciones de la & Q1 & $4.06(.79)$ & 85 & 251 & 396 & - 11 \\
\hline familia & Q3 & $4.15(.85)$ & -.85 & 251 & .396 & -0.11 \\
\hline Comunicación & & & & & & \\
\hline Me aseguro de que la familia haya entendido la información & Q1 & $4.38(.75)$ & 227 & 251 & 024 & 028 \\
\hline ofrecida en cada sesión & Q3 & $4.58(.65)$ & $|-2.27|$ & 201 & .024 & $\mid-0.28$ \\
\hline Adopto una actitud relajada en las conversaciones con la & Q1 & $4.41(.69)$ & A -261 & 252 & 010 & $-0,33$ \\
\hline familia & Q3 & $4.62(.55)$ & $\mid-2.61$ & & & \\
\hline
\end{tabular}


INTELIGENCIA EMOCIONAL Y PRÁCTICAS RELACIONALES CON LA FAMILIA EN ATENCIÓN TEMPRANA MARÍA MARCO ARENAS, MARÍA CRISTINA SÁNCHEZ LÓPEZ Y FRANCISCO ALBERTO GARCÍA SÁNCHEZ

\begin{tabular}{|c|c|c|c|c|c|c|}
\hline & $\mathrm{Q}$ & Media (dt) & $\mathrm{t}$ & $\mathrm{gl}$ & $\mathrm{p}$ & $\mathrm{d}$ \\
\hline \multicolumn{7}{|l|}{ Comunicación } \\
\hline \multirow{2}{*}{ Adapto mi lenguaje al nivel de comprensión de la familia } & Q1 & $4.71(.49)$ & \multirow{2}{*}{-2.12} & \multirow{2}{*}{251} & \multirow{2}{*}{.035} & \multirow{2}{*}{-0.26} \\
\hline & Q3 & $4.83(.42)$ & & & & \\
\hline \multicolumn{7}{|l|}{ Respeto hacia creencias y valores de la familia } \\
\hline \multirow{2}{*}{$\begin{array}{l}\text { Tengo en cuenta las opiniones de la familia sobre la } \\
\text { discapacidad }\end{array}$} & Q1 & $4.37(.76)$ & \multirow{2}{*}{-2.89} & \multirow{2}{*}{251} & \multirow{2}{*}{.004} & \multirow{2}{*}{-0.37} \\
\hline & Q3 & $4.62(.59)$ & & & & \\
\hline \multicolumn{7}{|l|}{ Reconocer habilidades y fortalezas en la familia } \\
\hline \multirow{2}{*}{$\begin{array}{l}\text { Analizo la disposición de la familia para participar en la } \\
\text { intervención }\end{array}$} & Q1 & $4.32(.79)$ & \multirow{2}{*}{-.819} & \multirow{2}{*}{252} & \multirow{2}{*}{.414} & \multirow{2}{*}{-0.1} \\
\hline & Q3 & $4.40(.85)$ & & & & \\
\hline
\end{tabular}

En la valoración que los profesionales hacen de las distintas propuestas de prácticas relacionales planteadas se encontraron 10 ítems en los que los profesionales con una habilidad de Reparación Emocional más baja (Q1) presentaban unas puntuaciones medias significativamente más bajas que sus compañeros con puntuaciones más altas en esa habilidad de inteligencia emocional (Q3). Esto supone que la habilidad de Reparación Emocional está afectando al $66.67 \%$ de los ítems de prácticas relacionales planteados.

En concreto, los profesionales situados en el tercer cuartil de Reparación Emocional puntúan significativamente más alto que sus compañeros del cuartil uno en tres ítems de la dimensión de prácticas relacionales denominada interacción entre profesional y familia ("me presento a la familia como un apoyo", "promuevo un clima de confianza en las interacciones con el cuidador principal" y "pregunto por el estado del niño al comenzar la sesión”); en tres ítems de la dimensión de trato a la familia ("soy sensible a las necesidades que manifiesta la familia", "identifico los distintos sentimientos o emociones que experimenta la familia" y "tengo un trato respetuoso hacia el entorno familiar”); en tres ítems de la dimensión de comunicación ("me aseguro de que la familia haya entendido la información ofrecida en cada sesión”, "adopto una actitud relajada en las conversaciones con la familia” y "adapto mi lenguaje al nivel de comprensión de la familia”); y en el ítem de la dimensión de respeto hacia las creencias y valores de la familia ("tengo en cuenta las opiniones de la familia sobre la discapacidad”). La habilidad de Reparación Emocional no ocasiona diferencias significativas en la dimensión de reconocimiento de babilidades y fortalezas en la familia. Los resultados de la prueba d de Cohen indican que el tamaño del efecto es, en todos los casos, entre pequeño y moderado. Obtiene el tamaño más bajo el ítem que plantea que el profesional tiene un trato respetuoso hacia el entorno familiar $(-0.25)$. Por su parte, el efecto alcanza un tamaño moderado en el ítem "promuevo un clima de confianza en las interacciones con el cuidador principal” (-0.58).

Confirmando el resultado ya apuntado para el primer objetivo del estudio, hay que señalar que se encontraron muchos ítems sobre prácticas relacionales con puntuaciones medias muy altas (ver Tablas 3, 4 y 5). En concreto, alcanzan medias por 
encima de 4.00 puntos (etiqueta verbal “Casi Siempre”) un total de 13 ítems $(86.67 \%)$ de profesionales del cuartil tres y 12 ítems (80\%) de profesionales del cuartil uno en las tres habilidades de inteligencia emocional valoradas. Por su parte, las medias de nueve ítems $(60 \%)$ de profesionales del cuartil tres superan el valor de 4.50 puntos, lo que supone acercarse a la etiqueta verbal de "Siempre" en la escala de valoración propuesta. Algo que solo ocurre en las medias de cuatro ítems (26.67\%) de los profesionales del cuartil uno en las habilidades de inteligencia emocional valoradas. Las puntuaciones más bajas las obtiene siempre el ítem de la dimensión Trato a la familia que es enunciado con la frase "juzgo la importancia de las preocupaciones de la familia”. Con unas medias algo mayores, pero también siempre por debajo de 4 puntos, queda también el ítem "Me adapto a las condiciones del entorno del niño sin modificarlo a mi estilo”, recogido en la dimensión de Interacción profesional-familia.

Por último, se quiso constatar el efecto o no de otras posibles variables en la valoración que los profesionales hacen de las prácticas relacionales desarrolladas con las familias con las que intervienen en Atención Temprana. Se analizaron, a partir de los datos sociodemográficos recogidos en el IPPAT, posibles diferencias debidas a la edad del profesional, su formación y su experiencia o veteranía en Atención Temprana. No se encontraron diferencias significativas entre las puntuaciones medias en prácticas relacionales de los profesionales de una edad de $\leq 30$ años frente a profesionales de $\geq$ 42 años $\left(\mathrm{t}_{(209)}=-1.11, \mathrm{p}=.268\right)$. Tampoco según la disciplina profesional del trabajador, agrupando disciplinas de ciencias sociales frente a ciencias sanitarias $\left(t_{(397)}=-0.40\right.$, $\mathrm{p}=.689$ ). De la misma forma, no se encontraron diferencias significativas en la valoración de las prácticas relacionales entre profesionales de $\leq 3$ años de experiencia laboral frente a profesionales veteranos de $\geq 14$ años en la disciplina $\left(\mathrm{t}_{(189)}=-.94, \mathrm{p}=.348\right)$.

\section{Discusión y conclusiones}

Las características de la muestra conseguida satisfacen nuestras expectativas. Se trata de un grupo de profesionales suficientemente amplio para los objetivos del estudio, donde están representados diferentes perfiles profesionales. Presentan un amplio rango de edades y encontramos, entre ellos, profesionales con diferente veteranía en Atención Temprana. Muchos de ellos suman formación específica en Atención Temprana a su titulación de base y su procedencia está repartida entre la mayoría de las comunidades autónomas del Estado español.

Respondiendo al primer objetivo del estudio, podemos concluir que todos los profesionales participantes llevan a cabo un importante volumen de prácticas relacionales con las familias que atienden. Prácticamente todos los ítems planteados reciben una valoración de que se hacen Siempre o Casi siempre. Este resultado era de esperar: el cuidado de las prácticas relacionales con la familia es un principio ampliamente demandado en la literatura científica de la disciplina, tanto nacional (Castellanos et al., 2003; Dalmau-Montala et al., 2017; GAT, 2000; Giné et al., 2008; Mendieta, 2005; Perpiñan, 2009), como internacional (Dempsy y Dunst, 2004; Dunst et al., 2002; Espe-Sherwindt, 2008; Sawyer y Campbell, 2012; Wilson y Dunst, 2005). Solo dos 
ítems obtienen puntuaciones que se quedan en la etiqueta verbal “A veces”. Pero precisamente son dos ítems que guardan una importante vinculación con el paradigma de intervención centrada en la familia. La filosofía de base de ese paradigma (Dunst et al., 2002; Espe-Sherwindt, 2008; García-Sánchez et al., 2014) lleva a que el profesional valore y considere continuamente las preocupaciones y prioridades de la familia, utilizando esa información para consensuar, con la familia, los objetivos inmediatos de intervención. A la vez, plantea que el profesional sea muy respetuoso para no modificar, imponiendo su estilo, las condiciones del entorno natural del niño. Seguramente, esta vinculación de esos ítems concretos a unas prácticas de Atención Temprana que aún están poco instauradas en nuestro país (Dalmay-Montala et al., 2017; García-Sánchez et al., 2014; Giné et al., 2008) ha contribuido a que queden puntuados con medias más bajas que el resto de ítems sobre prácticas relacionales.

Respondiendo a los objetivos segundo y tercero del estudio, nuestros resultados han permitido constatar que las habilidades de inteligencia emocional del profesional parecen estar influyendo en la valoración que hacen del volumen de prácticas relacionales que llevan a cabo en su intervención. No obstante, no se termina de detectar un patrón diferencial de influencia entre las tres habilidades de inteligencia emocional analizadas. Aunque parece que la habilidad de Reparación Emocional es la que influye en más ítems. Recordemos que esta habilidad emocional se define como la capacidad de afrontar y canalizar nuestros propios estados emocionales. Por otro lado, como respuesta al cuarto objetivo del estudio, se ha constatado que otras variables sociodemográficas no influyen en la valoración del volumen de prácticas relacionales realizadas. De acuerdo a estos resultados, podemos concluir que el volumen de prácticas relacionales que lleva a cabo el profesional con la familia efectivamente mantiene una vinculación con las habilidades de inteligencia emocional del profesional.

Para la obtención de los datos, hemos utilizado cuestionarios. Estos evalúan el metaconocimiento de las habilidades emocionales, por un lado, y de las prácticas relacionales realizadas, por el otro. Futuras investigaciones podrán utilizar, quizá, otras medidas más directas, por ejemplo, registros observacionales del quehacer del profesional. Pero lo cierto es que los resultados significativos encontrados son tan generalizados en la vinculación de prácticas relacionales realizadas con habilidades de inteligencia emocional que no pueden ser obviados sin más. Recordemos que hemos encontrado diferencias significativas en un rango que va del 40 al 67\% de los ítems sobre prácticas relacionales, según la habilidad emocional que analicemos.

Los puntos de corte utilizados en cada habilidad de inteligencia emocional para establecer los dos grupos de profesionales que hemos comparado en su valoración de las prácticas relacionales realizadas (cuartil 1 vs cuartil 3) nos hacen ver que los profesionales de Atención Temprana de nuestro estudio presentan buenos niveles de inteligencia emocional. Con todo, también encontramos cierto volumen de profesionales que tienen margen para mejorar en sus habilidades emocionales. Más en concreto, en la dimensión de Atención Emocional, el cuartil 1 quedaba delimitado en una puntuación de $\leq 21$ puntos. Según el manual de la TMMS-24, dicha puntuación indica claramente la necesidad de mejorar en esa habilidad, por prestar poca Atención Emocional. Por su parte, el cuartil 3 quedó delimitado por una puntuación de $\geq 28$. Se 
considera inadecuado, por prestar demasiada atención emocional, el superar valores de 32 puntos en mujeres y 35 en hombres. Pero esos valores no fueron alcanzados en nuestra muestra. Por su parte, en las habilidades de Claridad y Reparación emocional, las puntuaciones de los profesionales establecieron los cuartiles 1 y 3 en las puntuaciones medias $\leq 27$ y $\geq 34$, respectivamente. Según el manual del TMMS-24, estas habilidades deben mejorar cuando sus puntuaciones bajan de 23, son adecuadas a partir de ese valor y se adjetivan de excelentes a partir de valores de 35 o 36 puntos. Por tanto, la mayoría de los profesionales participantes presentan habilidades adecuadas e incluso excelentes de claridad y reparación emocional.

Futuras investigaciones deberán delimitar, con mayor precisión, ítems quizá más adecuados para valorar las diferentes dimensiones de las prácticas relacionales. $\mathrm{O}$ quizá también, como ya hemos apuntado, utilizar para su valoración registros observacionales directos del quehacer del profesional. Igualmente, futuras investigaciones deberán delimitar, con mayor precisión, las habilidades de inteligencia emocional que más pueden condicionar una buena práctica profesional en Atención Temprana. En cualquier caso, dado que esas habilidades pueden ser modificadas y ampliadas a través del aprendizaje, de su desarrollo y entrenamiento (Goleman, 1999; González, 2002; Qualter, Gardner y Whiteley, 2007; Teruel, 2000), nuestros resultados plantean la necesidad de integrar la formación en inteligencia y competencia emocional en el currículum del profesional de Atención Temprana. Más aún cuando, en los estudios realizados sobre necesidades formativas actuales de estos profesionales, se demanda claramente formación para mejorar capacidades relacionadas con la inteligencia emocional del profesional, como pueden ser la capacidad para el acompañamiento y desarrollo de competencias en la familia o para el trabajo en equipo (Rubio-Gómez, García-Sánchez y Orcajada, 2017). En este sentido, será siempre interesante incluir, en esa formación de los futuros profesionales, no solo la especificación de los contenidos y prácticas a aprender, sino también diferentes espacios de práctica, autoevaluación, reflexión, acompañamiento y feedback a través de supervisores/asesores cualificados (Dunst, 2015), los cuales deberían atender, también, el desarrollo de las habilidades emocionales de los futuros profesionales.

\section{Referencias bibliográficas}

Arnaldos, M. J., Casbas, M. I., Checa, F. J., Coloma, E., Funes, E., Galiana, R.... y Valero, J. (2000). Informe técnico sobre el modelo de intervención en Atención Temprana para la Región de Murcia. Revista de Atención Temprana, 3 (1), 37-47.

Azad, G., Blacher, J. y Marcoulides, G. A. (2013). Mothers of children with developmental disabilities: Stress in early and middle childhood. Research in Developmental Disabilities, 34 (10), 3449-3459. doi: 10.1016/j.ridd.2013.07.009.

BanerJee, R. y Luckner, J. (2014). Training needs of Early Childhood professional who work with children and families who are culturally and linguistically diverse. Infants and Young children, 27 (1), 43-59. doi: 10.1097/IYC.0000000000000000.

Barnett, D., Clements, M., Kaplan-Estrin, M. y Fialka, J. (2003). Building New Dreams. Infants and Young Children, 16 (3), 184-200. 
Bisquerra, R. y Pérez, N. (2007). Las competencias emocionales. Educación XXI, 10, 61-82.

Blue-Banning, M., Summers, J. A., Frankland, H. C., Nelson, L. L. y Beegle, G. (2004). Dimensions of family and professional partnerships: Constructive guidelines for collaboration. Council for Excepcional Children, 70 (2), 167-184.

Cantero, M. P. (2012). Competencias socioemocionales en la inserción laboral del egresado universitario (Tesis Doctoral). Universidad de Alicante. Alicante. Recuperado de http:// hdl.handle.net/10045/25132.

Castellanos, P., García-Sánchez, F. A., Mendieta, P., Gómez, L. y Rico, M. D. (2003). Intervención sobre la familia desde la figura del terapeuta-tutor del niño con necesidades de Atención Temprana. Siglo Cero, 34 (3), 5-18.

Côté, S. (2014). Emotional intelligence in organizations. Annual Review of Organizational Psychology and Organizational Behavior, 1, 459-488. doi: 10.1146/annurev-orgpsych$031413-091233$.

Côté, S. y Hideg, I. (2011). The ability to influence others via emotion displays: A new dimension of emotional intelligence. Organizational Psychology Review, 1, 53-71. doi:10.1177/2041386610379257.

Dalmau-Montala, M., Balcells, A., Giné, C., Cañadas, M., Casas, O., Salat, Y.... y CaLAF, N. (2017). Cómo implementar el modelo centrado en la familia en atención temprana. Anales de Psicología, 33 (3), 641-11. doi: 10.6018/analesps.33.3.263611.

DeMarle, D. J. y Le Roux, P. (2001). The life cycle and disability: experiences of discontinuity in child and family development. Journal of Loss and Trauma, 6, 29-43.

Dempsey, I. y Dunst, C. (2004). Helpgiving styles and parent empowerment in families with a young child with a disability. Journal of Intellectual and Developmental Disability, 29 (1), 40-51. doi: 10.1080/13668250410001662874.

Dunst, C. J. (2002). Family-centered practices: Birth through high school. The Journal of Special Education, 36 (3), 141-149.

Dunst, C. J. (2015). Improving the design and implementation of in-service professional development in Early Childhood Intervention. Infants and Young Children, 28 (3), 210-219. doi: $10.1097 /$ IYC. 0000000000000042 .

Dunst, C. J., Boyd, K., Trivette, C. M. y Hamby, D. W. (2002). Family-oriented program models and professional helpgiving practices. Family Relations, 51 (3), 221-229.

Dunst, C. J. y TrivetTe, C. M. (1996). Empowerment, effective helpgiving practices and family-centered care. Pediatric Nursing, 22, 334-337.

Escorcia, C. T., García-Sánchez, F. A., Sánchez-López, M. C. y Hernández-Pérez, E. (2016). Cuestionario de estilos de interacción entre padres y profesionales en atención temprana: validez de contenido. Anales de Psicología, 32 (1), 148-157. doi: 10.6018/analesps.32.1.202601.

ESPE-SHERWINDT, M. (2008). Family-centred practice: collaboration, competency and avidence. Support for Learning, 23 (3), 136-143. doi: 10.1111/j.1467-9604.2008.00384.x.

Espinoza, M., Sauhueza, O., Ramírez, N. y SÁez, K. (2015). Validación de constructo y confiabilidad de la escala de inteligencia emocional en estudiantes de enfermería. Revista LatinoAmericana de Enfermagem, 23 (1), 139-147.

Extremera, N. y Fernández-Berrocal, P. (2004). El uso de las medidas de habilidad en el ámbito de la inteligencia emocional. Ventajas e inconvenientes con respecto a las medidas de auto-informe. Boletín de Psicología, 80, 59-77.

Extremera, N., Fernández-Berrocal, P. y Durán, A. (2003). Inteligencia emocional y burnout en profesores. Encuentros en Psicología Social, 1, 260-265. 
INTELIGENCIA EMOCIONAL Y PRÁCTICAS RELACIONALES CON LA FAMILIA EN ATENCIÓN TEMPRANA MARÍA MARCO ARENAS, MARÍA CRISTINA SÁNCHEZ LÓPEZ Y FRANCISCO ALBERTO GARCÍA SÁNCHEZ

Fernández-Berrocal, P. (2010). La inteligencia emocional para médicos del siglo XXI. El Médico, 1112 (junio), 22-25.

Fernández-Berrocal, P. y Extremera, N. (2009). La inteligencia emocional y el estudio de la felicidad. Revista Interuniversitaria del Profesorado, 66 (23, 3), 85-108.

Fernández-Berrocal, P., Extremera, N. y Ramos, N. (2004). Validity and reliability of the Spanish modified version of the Trait Meta-Mood Scale. Psychological Reports, 94, 751-755.

Fernández-Zúñiga y Marcos de León, A. A. (2008). Habilidades terapéuticas en terapia de lenguaje. Relación terapeuta-paciente. Revista de Logopedia, Foniatría y Audiología, 28 (1), 34-45. doi: 10.1016/S0214-4603(08)70044-5.

García-Sánchez, F. A., Escorcia, C. T., Sánchez-López, M. C., Orcajada, N. y HernánDEZ, E. (2014). Atención temprana centrada en la familia. Siglo Cero, 45 (3), 6- 27.

GAT, Federación Estatal de Asociaciones de Profesionales de Atención Temprana (2000). Libro Blanco de la Atención Temprana. Madrid: Real Patronato sobre Discapacidad.

Gat (2011). La realidad actual de la Atención Temprana en España. Madrid: Real Patronato sobre Discapacidad.

Giné, C., Gràcia, M., Vilaseca, R. y Balcells, A. (2008). Trabajar con las familias en Atención Temprana. Revista Interuniversitaria de Formación del Profesorado, 65 (23, 2), 95-113.

Goleman, D. (1996). Inteligencia emocional. Barcelona: Kairós.

Goleman, D. (1999). La práctica de la inteligencia emocional. Barcelona: Kairós

GonZÁlEZ, V. (2002). ¿Qué significa ser un profesional competente? Reflexiones desde una perspectiva psicológica. Revista Cubana de Educación Superior, 22 (1), 45-53.

Jordan, P. y AshKANASY, N. M. (2006). Emotional intelligence, emotional self-awareness, and team effectiveness. En V. U. Druskat, F. Sala y G. Mount (eds.), Linking Emotional Intelligence and Performance at Work (pp. 145-163). Mahwah New Jersey: Lawrence Erlbaum Associates Inc.

Kaplan, J. E. y Welker, M. G. (2001). Team learning is here to stay: part 1 of the learning team series. New Accountant, 16 (3), 14-16.

King, G., Strachan, D., Tucker, M., Duwyn, M. B., Desserud, B. S. y Shillington, B. M. (2009). The application of a transdisciplinary model for Early Intervention services. Infants and Young Children, 22 (3), 211-223.

LOPEs, P. N. (2016). Emotional Intelligence in organizations: bridging research and practice. Emotion Review, 8 (4), 316-321. doi: 10.1177/1754073916650496.

Mendieta, P. (2005). Intervención familiar en Atención Temprana. En M. G. Millá y F. MuLAS (eds.), Atención Temprana. Desarrollo infantil, diagnóstico, trastornos e intervención (pp. 789-803). Valencia: Promolibro.

Peer, J. W. y Hillman, S. B. (2014). Stress and resilience for parents of children with intellectual and developmental disabilities: a review of key factors and recommendations for practitioners. Journal of Policy and Practice in Intellectual Disabilities, 11 (2), 92-98.

Qualter, P., Gardner, K. y Whiteley, H. (2007). Emotional Intelligence: review of research and educational implications. Pastoral Care in Education, 25 (1), 11-20. doi: 10.1111/ j.14680122.2007.00395.x.

Rubio-Gómez, N., García-Sánchez, F. A. y Orcajada, N. (2017, mayo). Estudio de necesidades formativas en prácticas de Atención Temprana centradas en la familia. En III Jornadas Doctorales de la Universidad de Murcia. Murcia.

SAwyer, B. y CAmpell, P. (2012). Early Interventionists' perspectives on teaching caregivers. Journal of Early Intervention, 34 (2), 104-124. 
Stoner, J., Meadan, H. y Angell, M. (2013). A model for coaching parents to implement teaching strategies with their young children with language delay or developmental disabilities. Perspectives on Language Learning and Education, 20, 112-119.

Teruel, M. P. (2000). La inteligencia emocional en el currículo de la formación inicial de los maestros. Revista Interuniversitaria de Formación del Profesorado, 38, 141-152.

Turnbull, A. P., Turnbull, H. R., Erwin, E. y Soodak, L. (2006). Families, professionals, and exceptionality. Positive outcomes though partnership and trust (5. ${ }^{\mathrm{a}}$ ed.). Columbus, $\mathrm{OH}$ y Upper Saddle River, NJ: Pearson/Merrill-Prentice Hall.

Wilson, L. y Dunst, C. J. (2005). Checklist for assessing adherence to family-centered practices. Case Tools, 1 (1), 1-6. 


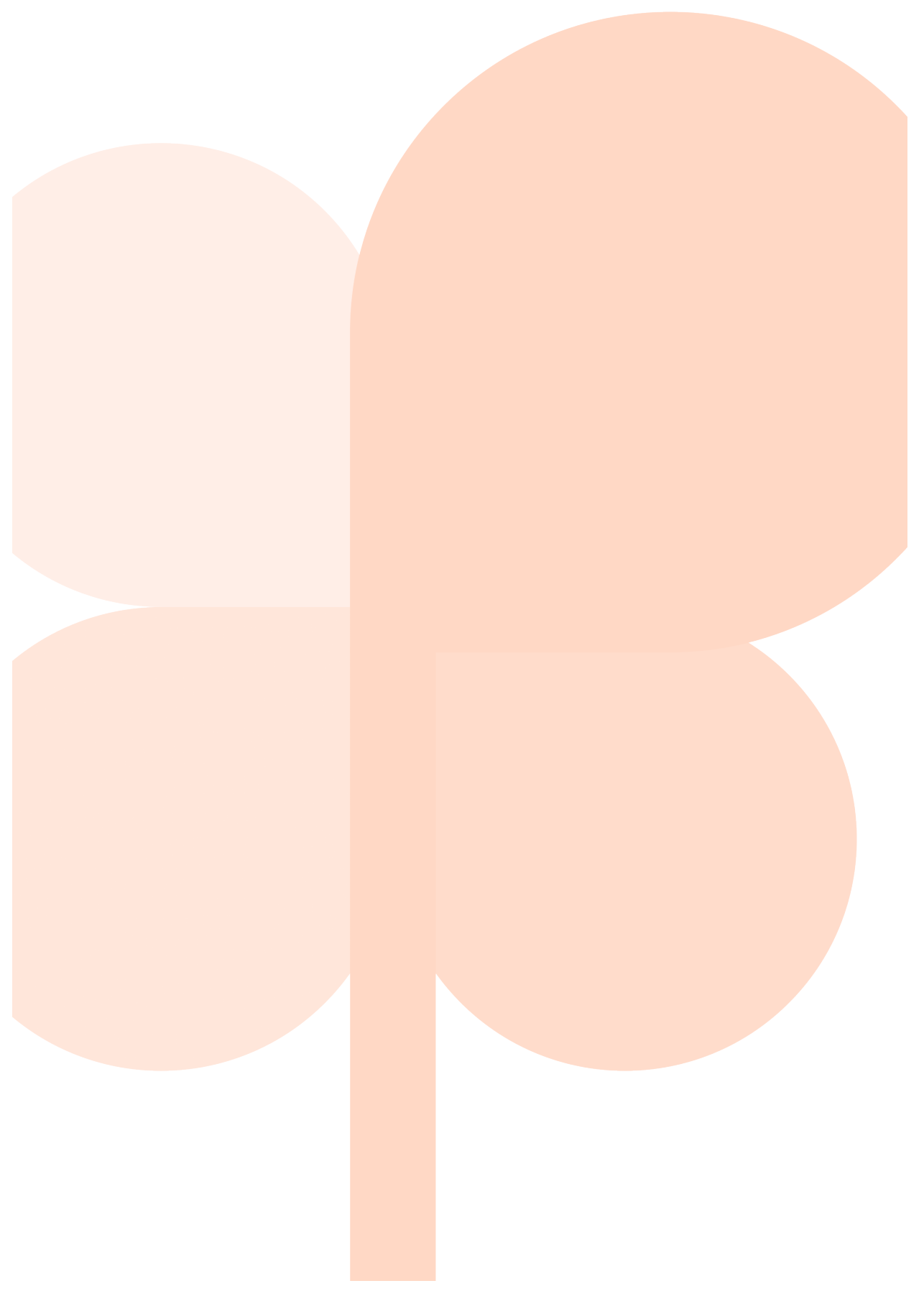

